\title{
Silica-Like Sequence of Anomalies in Core-Softened Systems
}

\author{
Yu. D. Fomin and E. N. Tsiok \\ Institute for High Pressure Physics, Russian Academy of Sciences, Troitsk 142190, Moscow Region, Russia \\ V. N. Ryzhov \\ Institute for High Pressure Physics, Russian Academy of Sciences, Troitsk 142190, Moscow Region, Russia and \\ Moscow Institute of Physics and Technology, 141700 Moscow, Russia
}

(Dated: July 1, 2018)

\begin{abstract}
In this paper we present a simulation study of density, structural and diffusion anomalies in coresoftened system introduced in our previous publications. It is well-known, that with appropriate parametrization, core-softened systems are remarkable model liquids that exhibit anomalous properties observed in tetrahedral liquids such as silica and water. It is widely believed that core-softened potentials demonstrate the water-like sequence of anomalies. We show that with increasing the depth of the attractive part of the potential the order of the region of anomalous diffusion and the regions of density and structural anomalies is inverted and have the silica-like sequence. We also show that the slope of the Widom line is negative like in water.
\end{abstract}

PACS numbers: 61.20.Gy, 61.20.Ne, 64.60.Kw

In the last decades there is a growing interest to a class of fluids that display anomalous thermodynamic and kinetic behaviors. The most common and well known example is water. The water phase diagram has regions where a thermal expansion coefficient is negative (density anomaly), self-diffusivity increases upon compression (diffusion anomaly), and the structural order of the system decreases with increasing pressure (structural anomaly) [1 $[6]$. Later on it was discovered that many other substances also demonstrate similar behavior. Some typical examples are silica, silicon, phosphorus and many others [5 10].

As it was found in experiments 1] and simulations [3, 4], the water anomalies have a well-defined sequence: the regions where these anomalies take place form nested domains in the density-temperature 3] (or pressuretemperature [4]) planes: the density anomaly region is inside the diffusion anomaly domain, and both of these anomalous regions are inside a broader structurally anomalous region. This water-like behavior was found in systems with spherically symmetric core-softening potentials with two length scales [11 27].

However, in other anomalous systems the sequence of anomalies may be different. For example, in computer simulation of the system with the Van Beest-Kramer-Van Santen (BKS) potential the hierarchy of anomalies for silica is different compared to water 7]. In this case the diffusion anomaly region contains the structural anomalous region which, in turn, has the density anomaly region inside. To our knowledge this is the only example of such inversion of the order of the anomalies discussed in literature till now.

It is widely believed that in the core-softened systems hierarchy of anomalies has the water-like type. For example, Yan et al. [17, 18] characterized the structural, thermodynamic, and kinetic properties of a family of discon- tinuous core-softened potentials that vary in the length scale of the soft repulsion region. In each case, they found the same relationship between anomalous regions as observed for water: structural anomalies preceded diffusivity anomalies, which preceded density anomalies. In Ref. 19] it was discussed an approach for the analysis of the anomalous behavior based on the well known Rosenfeld scaling relations [28] which connect the transport coefficients with excess entropy. In this case there was obtained the explicit equation determining the appearance of the anomalies in system [19]:

$$
\left(\frac{\partial S_{e x}}{\partial \ln \rho}\right)_{T}>c
$$

where $\rho$ is the number density, $S_{e x}=S-S_{i d}$ is the excess entropy, equal to difference between total $S$ and ideal gas $S_{i d}$ entropies, and $c$ is the property-specific constant. Based on Rosenfeld's scaling parameters [19], it was shown that value $c=0$ corresponds to structural anomaly, $c=0.42$ to diffusion anomaly, and $c=1$ to density anomaly. From (1) one can conclude that anomalous behavior always occur in the water-like order: structural anomalies precede diffusivity anomalies, which in turn precede density anomalies. This conclusion was verified by the computer simulation of the system interacting through a two-scale potential introduced by Jagla 29]. However, as it was shown in our previous publications [23, 25], Rosenfeld scaling fails in the vicinity of anomalies, and it can not be used for the correct analysis of the order of the anomalies.

It is important to note that the equation (1) contains two conditions which are basing on strict thermodynamic arguments: the conditions for structural and density anomalies [19]. This means that the density anomaly should always follow after the structural anomaly. On the other hand, the diffusion anomaly can be located at 


\begin{tabular}{|c|c|c|c|c|c|c|}
\hline number & $\sigma_{1}$ & $\sigma_{2}$ & $\lambda_{0}$ & $\lambda_{1}$ & $\lambda_{2}$ & well depth \\
\hline 1 & 1.35 & 0 & 0.5 & 0.5 & 0 & 0 \\
2 & 1.35 & 1.80 & 0.5 & 0.60 & 0.10 & 0.20 \\
3 & 1.35 & 1.80 & 0.5 & 0.7 & 0.20 & 0.4 \\
\hline
\end{tabular}

TABLE I: The potential parameters used in simulations (Eq. (2)).

every place. For example, for silica the diffusion anomaly precedes the structural and density anomalies.

This paper presents a simulation study of anomalies in core-softened system introduced in our previous publications [20 27]. We investigate the sequence of the anomalous regions and find that with increasing the attractive part of the potential the system demonstrates both water-like and silica-like behavior. It is also shown that the potential qualitatively correctly reproduces the behavior of the Widom line for the liquid-liquid transition.

In the present study we investigate a system of particles interacting via the potential with "hard" core, repulsive shoulder and attractive well [21, 25].

The general form of the potential is written as

$$
\begin{aligned}
U(r) & =\varepsilon\left(\frac{\sigma}{r}\right)^{14}+\varepsilon\left(\lambda_{0}-\lambda_{1} \tanh \left(k_{1}\left\{r-\sigma_{1}\right\}\right)+\right. \\
& \left.+\lambda_{2} \tanh \left(k_{2}\left\{r-\sigma_{2}\right\}\right)\right)
\end{aligned}
$$

Here $k_{1}=k_{2}=10.0$ and the parameters of the potentials (2) are given in Table 1. The family of the potentials with $\sigma_{1}=1.35$ and different attractive wells is shown in Fig. 1. It should be noted that the potential (2) is very similar to the Fermi-Jagla potential suggested recently in Ref. [32], where the Fermi distribution function is used except for the hyperbolic tangent in Eq. (2) in order to describe the smoothed step [33].

In the remainder of this paper we use the dimensionless quantities: $\tilde{\mathbf{r}} \equiv \mathbf{r} / \sigma, \tilde{P} \equiv P \sigma^{3} / \varepsilon, \tilde{V} \equiv V / N \sigma^{3} \equiv 1 / \tilde{\rho}, \tilde{T} \equiv$ $k_{B} T / \varepsilon$. As we will only use these reduced variables, we omit the tildes.

In Refs. [20 27] it was shown that these systems demonstrate anomalous behavior. A relation between phase diagram and anomalous regions was also discussed in these articles. Our later publications gave detailed study of diffusion, density and structural anomalies in this system [23 27].

In the present article we carry out a molecular dynamics and Monte Carlo study of the core-softened systems and monitor the change in phase diagram and anomalous regions with changing the potential parameters. The details of simulations can be found in Ref. [26].

In order to find the melting lines we carry out the free energy calculations for different phases and construct a common tangent to them. For our potentials we computed the free energy of the liquid by integrating the equation of state along an isotherm [34]: $\frac{F(\rho)-F_{i d}(\rho)}{N k_{B} T}=$

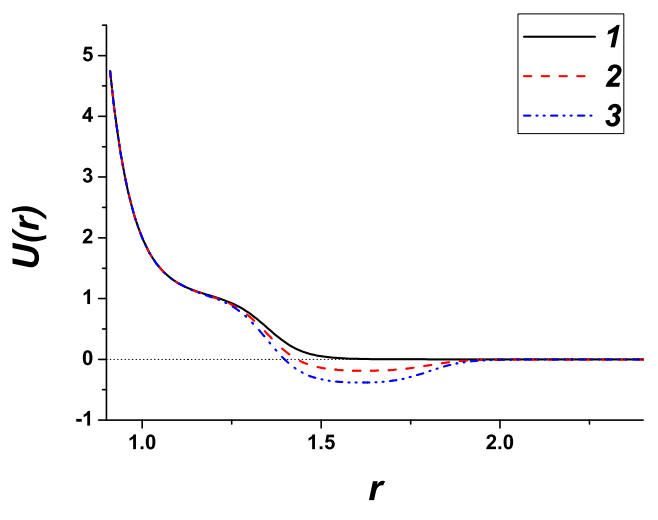

FIG. 1: (Color online) Family of the potentials with $\sigma_{1}=1.35$ and different attractive wells. The curves are numerated in accordance with Table 1.

$\frac{1}{k_{B} T} \int_{0}^{\rho} \frac{P\left(\rho^{\prime}\right)-\rho^{\prime} k_{B} T}{\rho^{\prime 2}} d \rho^{\prime}$. Free energies of different crystal phases were determined by the Monte Carlo simulations with the method of coupling to the Einstein crystal [34]. In this case the excess entropy can be computed via $S_{e x}=\frac{U-F_{e x}}{N k_{B} T}$, where $U$ is the internal energy [34]. The total entropy is $S=S_{e x}+S_{i d}$, where the ideal gas entropy is $\frac{S_{i d}}{N k_{B}}=\frac{3}{2} \ln (T)-\ln (\rho)+\ln \left(\frac{\left(2 \pi m k_{B}\right)^{3 / 2} e^{5 / 2}}{h^{3}}\right)$.

Here we present the anomalous regions for the three systems shown in Fig. 1

The density anomaly means that density increases upon heating or that the thermal expansion coefficient becomes negative. Using the thermodynamic relation $(\partial P / \partial T)_{V}=\alpha_{P} / K_{T}$, where $\alpha_{P}$ is a thermal expansion coefficient and $K_{T}$ is the isothermal compressibility and taking into account that $K_{T}$ is always positive and finite for systems in equilibrium not at a critical point, we conclude that density anomaly corresponds to minimum of the pressure dependence on temperature along an isochor. This is the most convenient indicator of density anomaly in computer simulation.

Initially the structural anomaly was introduced via order parameters characterizing the local order in liquid [3, 7, 16, 17, 37 39]. However, later on the local order was also related to excess entropy of the liquid which is defined as the difference between the entropy and the ideal gas entropy at the same $(\rho, T)$ point: $S_{e x}=S-S_{i d}$. In normal liquid excess entropy is monotonically decaying function of density along an isotherm while in anomalous liquids it demonstrates increasing in some region. This allows to define the boundaries of structural anomaly at given temperature as minimum and maximum of excess entropy.

The behavior of the diffusion coefficient, pressure and excess entropy for the purely repulsive system with $\sigma_{1}=$ 1.35 has been discussed, for example, in Refs. 20, 23, 2527]. One can see that all three anomalies take place in the system. Fig. 2(a) places the regions of the anomalies 
in the phase diagram. As it was shown in [20, 21, 26] (see, for example, Fig. 1 in Ref. 21]), the phase diagram of the system consists of the high density and low density Face Centered Cubic (FCC) phases, corresponding to hard core and repulsive shoulder parts of the potential, separated by a sequence of crystalline phases. In Fig. 2(a) we show the low density part of the phase diagram with the FCC and FCT (Face Centered Tetragonal) phases.

One can see that the anomalous regions correspond to the picture proposed for water [3], i.e. the diffusion anomaly region is inside the structural anomaly and the density anomaly is mainly inside the diffusion anomaly.

In Ref. [26] it was shown that in the system with the purely repulsive potential the diffusion and density anomalies inverted with respect to each other with increasing the repulsive core diameter, i.e. now diffusion anomaly region is inside the density anomaly one.

Next we consider the influence of attraction on the anomalous behavior of the system. For this, we study the system with step size $\sigma_{1}=1.35$ and different well depths (Table 1 and Fig. 1). In this case one can see that with increasing the depth of the attractive well the sequence of the anomalies also inverted: for $w=0$ we have the waterlike order of the anomalies (see Fig. $2(\mathrm{a})$ ), at $w=0.2$ the locations of the diffusion and structural anomalies almost coincide (Fig. 2(b)). At last, for $w=0.4$ the diffusion anomaly region contains the structural anomalous region which, in turn, has the density anomaly region inside (Fig. 2(c)) [40]. As a result, for $w=0.4$ we obtain the configuration of anomalies which is the same as in silica 7].

It seems that a most popular point of view is that the hypothesized liquid-liquid critical point is the thermodynamic source of all water anomalies [31, 41 43], the terminal point of a line of first-order liquid-liquid phase transition. The line emanating from this critical point is sometimes called the Widom line and is often considered as an extension of the coexistence line into the onephase region (see, for example, [31, 32, 45]). This line is determined by the lines of the maxima of the thermodynamic response functions which asymptotically approach one another as the critical point is approached [31, 45]. The lines of the liquid-liquid phase transition in the computer simulations of water [31] and silicon [44] have the negative slope. On the other hand, earlier computer simulations of the isotropic core-softened potentials (see, for example, [31]) suggested that the slope of the Widom line is positive in these systems.

In Fig. (3) we show the maxima lines of isobaric heat capacity $C_{P}$ along isobars and isotherms, the line of the isothermal compressibility maxima $K_{T}$, the line of the thermal expansion coefficient $\alpha_{P}$ minima along with the melting line for the systems 2 and 3 (Table 1 ). One can see, that for our potential the Widom line has a slope which coincides with the results for water [31] and silicon
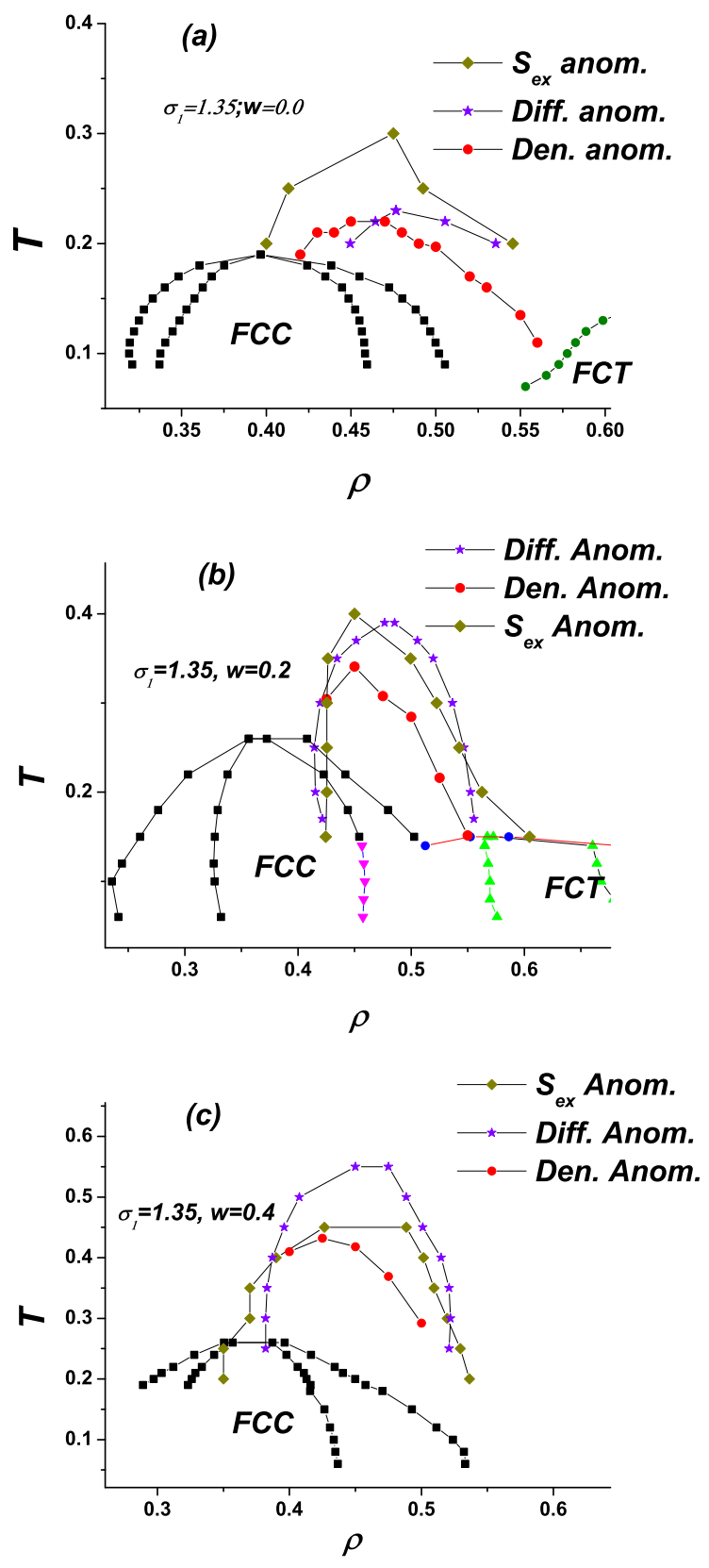

FIG. 2: (Color online) (a) Location of anomalous regions at the low density part of the phase diagram of the system with $\sigma_{1}=1.35$, where Face Centered Cubic (FCC) and Face Centered Tetragonal (FCT) phases are shown. The water-like order of anomalies takes place: the density anomaly region is inside the diffusion anomaly domain, and both of these anomalous regions are inside a broader structurally anomalous region. Location of anomalous regions at the phase diagram for (b) system with $\sigma_{1}=1.35$ and $w=0.2$; (c) $\sigma_{1}=1.35$ and $w=0.4$ with the silica-like order of anomalies (see Table $1)$. 

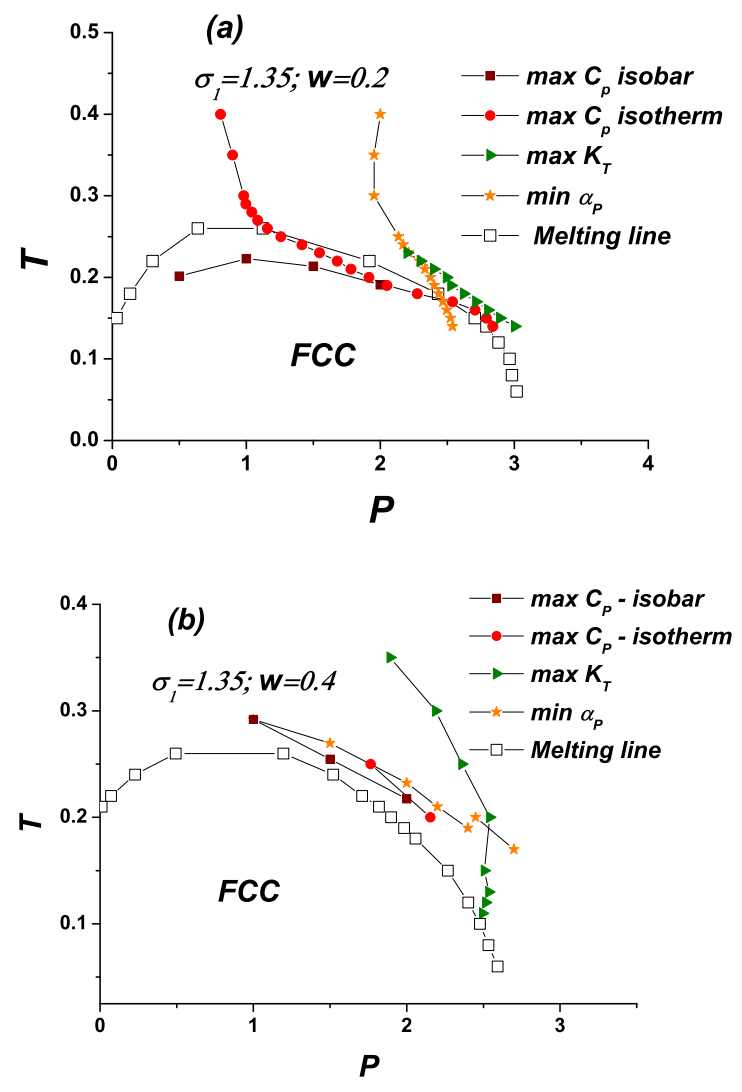

FIG. 3: (Color online) The maxima lines of isobaric heat capacity $C_{P}$ along isobars and isotherms, the line of the isothermal compressibility maxima $K_{T}$, the line of the thermal expansion coefficient minima along with the melting line for the systems 2 (a) and 3 (b) (Table 1).

[44]. It should be noted that the similar results were obtained recently [32] for the potential similar to (2).

In conclusion, this publication represents a detailed computer simulation study of anomalous behavior of core-softened systems proposed in our publications 20 27]. The core-softened potentials are widely investigated because they reproduce the water-like anomalies. Taking into account that the anomalies also exist in the systems where the hydrogen bonds are absent, it seems that the unusual properties of water are quite universal and can be investigated with the help of the isotropic core-softened potentials. To our knowledge, all isotropic core-softened potentials which were considered in the previous publications show the sequence of anomalies characteristic for water: the density anomaly region is inside the diffusion anomaly domain, and both of these anomalous regions are inside a broader structurally anomalous region. On the other hand, in other anomalous systems the sequence of anomalies may be different. For example, the hierarchy of anomalies for silica is different compared to water. In the present article we analyze the possibility of changing
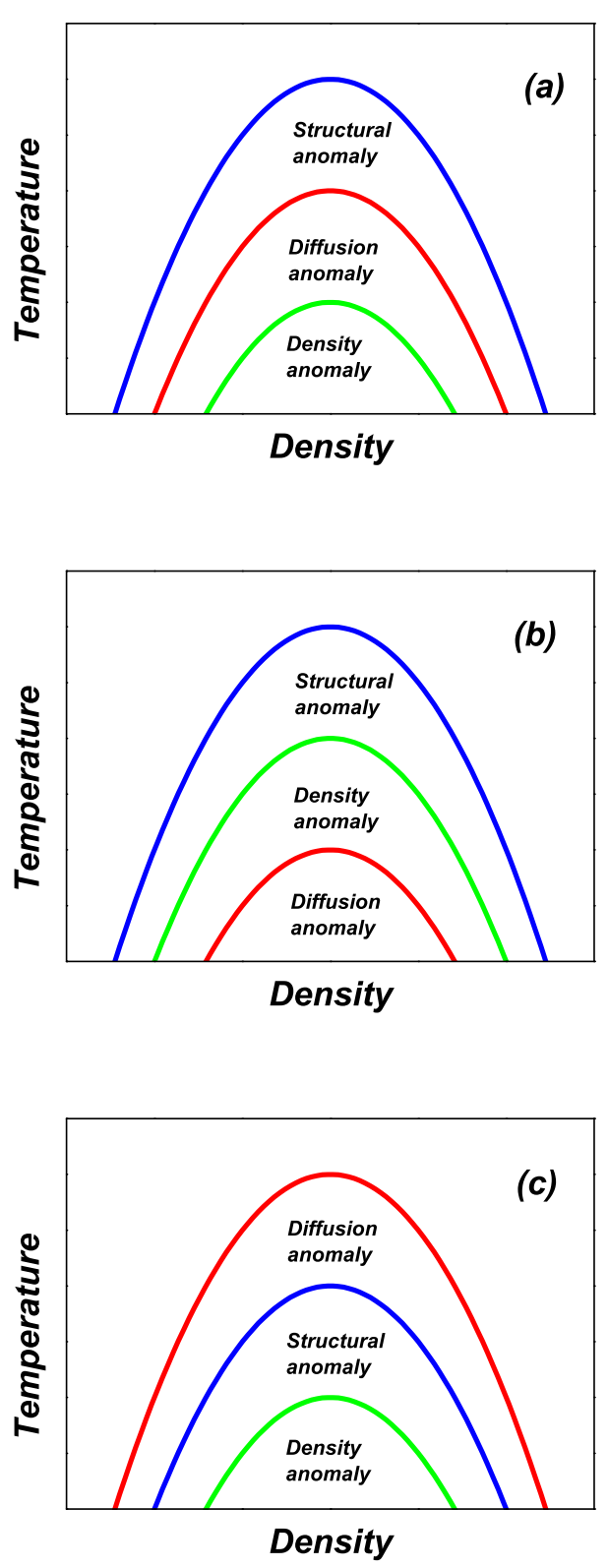

FIG. 4: (Color online) Schematic of the regions wherein structural, diffusivity, and density anomalies are found within the temperature-density planes. Region of anomalous density behavior always appears as nested dome within the structural anomalous envelope while the diffusivity anomaly may be (a) between the structural and density ones (see Fig. 2 (waterlike behavior); (b) inside the density anomalous region; and (c) the outermost envelope (silica-like behavior).

the order of anomalies regions depending on the parameters of the potential. It is shown that for the potential (2) for small values of the repulsive step the sequence of anomalies is the same as in water, however, with increasing the width of the repulsive shoulder the order of the region of anomalous diffusion and the region of den- 
sity anomaly is inverted. With increasing the depth of the attractive well we obtain the configuration of anomalies which is the same as in silica [7], where the diffusion anomaly region contains the structural anomalous region which, in turn, has the density anomaly region inside. It seems that this is the first case when the isotropic coresoftened system demonstrates the sequence of anomalies which is different from the water one and may be the same as in silica. It is also shown that for the potential (2) the slope of the Widom line is negative as in the case of water and silicon.

We thank S. M. Stishov and V. V. Brazhkin for stimulating discussions. Y.F. and E.T. also thanks Russian Scientific Center Kurchatov Institute and Joint Supercomputing Center of Russian Academy of Science for computational facilities. The work was supported in part by the Russian Foundation for Basic Research (Grants No 11-02-00341-a, 13-02-00579-a, and 13-02-00913-a) and the Ministry of Education and Science of Russian Federation projects 8370 and 8512 .

[1] C.A. Angell, E.D. Finch, and P. Bach, J. Chem. Phys. 65, 3063 (1976).

[2] O. Mishima and H. E. Stanley, Nature 396, 329 (1998).

[3] J. R. Errington and P. G. Debenedetti, Nature (London) 409, 318 (2001).

[4] P.A. Netz, F.V. Starr, H.E. Stanley, and M.C. Barbosa, J. Chem. Phys. 115, 318 (2001).

[5] P. G. Debenedetti, Metastable Liquids: Concepts and Principles (Princeton University Press, Princeton, 1998).

[6] V. V. Brazhkin. S. V. Buldyrev, V. N. Ryzhov, and H. E. Stanley [eds], New Kinds of Phase Transitions: Transformations in Disordered Substances [Proc. NATO Advanced Research Workshop, Volga River] (Kluwer, Dordrecht, 2002).

[7] M.S. Shell, P.G. Debenedetti, A.Z. Panagiotopoulos, Phys. Rev. E 66, 011202 (2002).

[8] V.V. Brazhkin, E.L. Gromnistkaya, O.V. Stalgorova, A.G. Lyapin, Rev. High Pressure Sci. Technol. 7, 1129 (1998).

[9] Y. Katayama, T. Mizutani, W. Utsumi, O. Shimomura, M. Yamakata, K. Funakoshi, Nature (London) 403, 170 (2000).

[10] Y. Katayama, Y. Inamura, T. Mizutani, W. Yamakata, M. Utsumi, S.O., Science 306, 848 (2004).

[11] Pol Vilaseca and Giancarlo Franzese, Journal of NonCrystalline Solids 357, 419 (2011).

[12] S. V. Buldyrev, G. Malescio, C. A. Angell, N. Giovambattista, S. Prestipino, F. Saija, H. E. Stanley and L. Xu, J. Phys.: Condens. Matter 21, 504106 (2009).

[13] Pol Vilaseca and Giancarlo Franzese, J. Chem. Phys., 133, 084507 (2010).

[14] G. Franzese, J. Mol. Liq. 136, 267 (2007).

[15] A. B. de Oliveira, G. Franzese, P. A. Netz, and M. C. Barbosa, J. Chem. Phys. 128, 064901 (2008).

[16] A. B. de Oliveira, P. A. Netz, T. Colla, and M. C. Bar- bosa, J. Chem. Phys. 125, 124503 (2006).

[17] Z. Yan, S. V. Buldyrev, N. Giovambattista, and H. E. Stanley, Phys. Rev. Lett. 95, 130604 (2005).

[18] Z. Yan, S. V. Buldyrev, N. Giovambattista, P. G. Debenedetti, and H. E. Stanley, Phys. Rev. E 73, 051204 (2006).

[19] J. R. Errington, Th. M. Truskett, J. Mittal, J. Chem. Phys. 125, 244502 (2006).

[20] Yu. D. Fomin, N. V. Gribova, V. N. Ryzhov, S. M. Stishov and Daan Frenkel, J. Chem. Phys., 129, 064512 (2008).

[21] Yu. D. Fomin, E.N. Tsiok and V.N. Ryzhov, J. Chem. Phys. 134, 044523 (2011).

[22] N. V. Gribova, Yu. D. Fomin, Daan Frenkel, V. N. Ryzhov, Phys. Rev. E 79, 051202 (2009).

[23] Yu. D. Fomin, V. N. Ryzhov, N. V. Gribova, Phys. Rev. E 81, 061201 (2010).

[24] Yu. D. Fomin and V. N. Ryzhov, Phys. Lett. A 375, 2181 (2011).

[25] Yu. D. Fomin, E.N. Tsiok and V.N. Ryzhov, J. Chem. Phys. 135, 124512 (2011).

[26] Yu. D. Fomin, E. N. Tsiok, and V. N. Ryzhov, J. Chem. Phys. 135, 234502 (2011).

[27] Yu. D. Fomin, E. N. Tsiok, and V. N. Ryzhov, European Physical Journal - Special Topics 216, 165 (2013).

[28] Ya. Rosenfeld, J. Phys.: Condens. Matter 11, 5415 (1999).

[29] E. A. Jagla, J. Chem. Phys. 111, 8980 (1999).

[30] E. A. Jagla, Phys. Rev. E 63, 061509 (2001).

[31] L. Xu, P. Kumar, S.V. Buldyrev, S.-H. Chen, P.H. Poole, F. Sciortino, and H.E. Stanley, PNAS 102, 16558 (2005).

[32] J.A. Abraham, S.V. Buldyrev, and N. Giovambattista, J. Phys. Chem. B 115, 14229 (2011).

[33] Using the identity $n_{F}(x)=\frac{1}{2}\left\{1-\tanh \frac{x}{2}\right\}$, where the Fermi-function, $n_{F}(x)=1 /[1+\exp (x)]$, one can easily rewrite the potential (2) in the form used in 32].

[34] Daan Frenkel and Berend Smit, Understanding molecular simulation (From Algorithms to Applications), 2nd Edition (Academic Press), 2002.

[35] E. A. Koopman and C.P. Lowe, J. Chem. Phys. 124, 204103 (2006).

[36] A.B. de Oliveira, P.A. Netz and M.C. Barbosa, Europhys. Lett. 85, 36001 (2009).

[37] J. R. Errington, P. G. Debenedetti, and S. Torquato, J. Chem. Phys. 118, 2256 (2003).

[38] P. J. Steinhardt, D. R. Nelson, and M. Ronchetti, Phys. Rev. B 28, 784 (1983).

[39] S. Torquato, T. M. Truskett, and P. G. Debenedetti, Phys. Rev. Lett. 84, 2064 (2000).

[40] We would like to mention, that in our previous paper 25] there is an error in Fig.17(c), and it should be replaced by the present Fig. 2(c).

[41] P. H. Poole, F. Sciortino, U. Essmann, H. E. Stanley, Nature 360, 324 (1992).

[42] O. Mishima, H. E. Stanley, Nature 392, 164 (1998).

[43] G. Franzese, G. Malescio, A. Skibinsky, S. V. Buldyrev, H. E. Stanley, Nature 409, 692 (2001).

[44] V. V. Vasisht, S. Saw, and S. Sastry, Nature Physics 7, 549 (2011).

[45] V. V. Brazhkin and V. N. Ryzhov, J. Chem. Phys. 135, 084503 (2011). 